\title{
Cavitation inception by almost spherical solid particles in water
}

\author{
Marschall, H.B.; Mørch, Knud Aage; Keller, A.P.; Kjeldsen, M.
}

Published in:

Physics of Fluids

Link to article, DOI:

$10.1063 / 1.1535940$

Publication date:

2003

Document Version

Publisher's PDF, also known as Version of record

Link back to DTU Orbit

Citation (APA):

Marschall, H. B., Mørch, K. A., Keller, A. P., \& Kjeldsen, M. (2003). Cavitation inception by almost spherical solid particles in water. Physics of Fluids, 15(2), 545-553. https://doi.org/10.1063/1.1535940

\section{General rights}

Copyright and moral rights for the publications made accessible in the public portal are retained by the authors and/or other copyright owners and it is a condition of accessing publications that users recognise and abide by the legal requirements associated with these rights.

- Users may download and print one copy of any publication from the public portal for the purpose of private study or research.

- You may not further distribute the material or use it for any profit-making activity or commercial gain

- You may freely distribute the URL identifying the publication in the public portal

If you believe that this document breaches copyright please contact us providing details, and we will remove access to the work immediately and investigate your claim. 


\title{
Cavitation inception by almost spherical solid particles in water
}

\author{
H. B. Marschall \\ Versuchsanstalt für Wasserbau Obernach, Munich University of Technology, D-82432 Obernach, Germany \\ K. A. Mørch \\ Department of Physics, Technical University of Denmark, DK-2800 Kgs. Lyngby, Denmark \\ A. P. Keller \\ Versuchsanstalt für Wasserbau Obernach, Munich University of Technology, D-82432 Obernach, Germany \\ M. Kjeldsen \\ Department of Thermal Energy and Hydropower, Norwegian University of Science and Technology, \\ N-7043 Trondheim, Norway
}

(Received 21 May 2002; accepted 14 November 2002; published 8 January 2003)

\begin{abstract}
The tensile strength of water increases when solid particles are filtered out, and it becomes greater the smaller the remaining particles are. Natural particles are of random shape, making parametric studies on the relationship between tensile strength and particle characteristics difficult. In this investigation, using degassed tap water from which natural particles larger than about $1 \mu \mathrm{m}$ had been filtered out, the tensile strength was measured before and after seeding with almost spherical solid balls of diameters from 3 up to $76 \mu \mathrm{m}$. The smallest balls, one type being hydrophobic, the other hydrophilic, had no measurable influence on the tensile strength, though they were notably larger than the remaining natural nuclei. Seeding with the larger balls, hydrophilic as well as hydrophobic ones, reduced the tensile strength compared with that measured for unseeded, filtered water, but at most down to $1 / 3$. On this basis it is concluded that a greater tensile strength is connected to the almost spherical solid balls than that due to natural particles of the same size. The critical cavities developed from the larger balls had radii much smaller than those of the balls themselves. This supports the hypothesis that cavitation nuclei are related to the fine scale surface structures observed on the balls, and in dependence of their global radii of curvature, critical cavities are developed. A model of this development is presented. (C) 2003 American Institute of Physics.
\end{abstract} [DOI: $10.1063 / 1.1535940]$

\section{INTRODUCTION}

The ability of liquids to sustain tensile stress is normally limited to relatively small negative pressures due to the occurrence of cavitation. The change from a one-phase liquid to a two-phase bubbly medium is ascribed to the presence of cavitation nuclei which grow unstably at exposure of the liquid to a sufficient tensile stress. The nature of these cavitation nuclei has been the subject of continuous discussion in the cavitation community. Gas bubbles embedded in the bulk of a liquid have been suggested, but they dissolve ${ }^{1}$ or disappear due to buoyancy unless they are somehow stabilized. Harvey et al. ${ }^{2}$ proposed that gas nuclei are stabilized in hydrophobic conical cracks and crevices of solid surfaces. Though solid surfaces generally do not exhibit such features on a submicrometer scale, the model proposed by Harvey et al. is able to explain several experimental observations of cavitation inception. Greenspan and Tschiegg ${ }^{3}$ showed that the removal of particles larger than $0.2 \mu \mathrm{m}$ diameter from water increased the tensile strength to about 200 bar. This supports the supposition that the free stream nuclei causing cavitation are closely connected to the solid particles always abundantly present in plain water. Recently it was suggested in a revised Harvey-type model ${ }^{4}$ that liquids may detach from solid surfaces at localities of concave curvature so that interfacial voids are formed. This is ascribed to interfacial liquid tension and to the diffusion of gas molecules into the structured interfacial liquid. Under certain conditions the voids may develop into larger stabilized cavitation nuclei because sound fields in the liquid set up broadband resonance vibrations of the gas-liquid interfaces. In such cases rectified diffusion of gas into the voids balances the diffusion out of them due to the excess pressure associated with their convex gas-liquid interfaces. Of course, the degree of gas saturation in the liquid at the prevailing pressure also strongly influences this diffusion balance.

Large natural particles are generally of highly irregular shape and their surfaces exhibit a broad spectrum of local radii of curvature (apparent from Crum's Fig. $1^{5}$ ). Attached gaseous voids are expected to develop at these irregularities, and their growth at exposure to tensile stress determines the tensile strength $P_{\text {ts }}$ of the liquid. In order to study how the tensile strength is dependent on the particle shape and surface character, it is advantageous to replace the natural particles with well-known ones. In this paper experiments with monosized almost spherical particles of different diameters and wettabilities are described. 


\section{SPHERICAL SOLID PARTICLES}

The tensile strength of a liquid increases if solid particles are removed by filtering - the more the smaller the remaining particles are. If the liquid is subsequently seeded with perfectly spherical particles of radius $R$, which are totally hydrophobic, the theoretically smallest possible tensile strength $\left(P_{\mathrm{ts}}\right)_{R \text {,th }}$ is determined from the stress $p_{\infty}=p_{\infty}$, crit that overcomes the surface tension force and makes a vapor cavity of initial radius $R$ detach from the solid surface and grow, i.e.,

$$
\left(P_{\mathrm{ts}}\right)_{R, \mathrm{th}}=2 \gamma / R=-\left(p_{\infty}-p_{v}\right)_{\mathrm{crit}},
$$

in which $\gamma$ is the surface tension constant and $p_{v}$ is the vapor pressure. Thus, a totally hydrophobic spherical particle $R=1.5 \mu \mathrm{m}$ in cold water results in $\left(P_{\mathrm{ts}}\right)_{R, \mathrm{th}} \approx 1.0$ bar. (Actually, perfectly hydrophobic solids do not exist due to the occurrence of van der Waals' forces, but a $100 \%$ hydrophobic sphere equals a vapor bubble.) In cases of partially hydrophobic spheres the tensile strength is greater because liquid-solid bonds have to be broken. Hydrophilic, perfect spheres are not responsible for the tensile strength because the molecules of the liquid bond better to the sphere than to neighboring liquid molecules. However, real particles of spherical shape exhibit small-scale deviations from sphericity, so that locally concave surface structures are formed. At such localities interfacial gaseous voids - cavitation nucleimuch smaller than the particles themselves may be in dynamic equilibrium. ${ }^{4}$ By pressure reduction these voids grow, and adjacent ones may merge into a single, larger one. In a rapid pressure reduction the content of noncondensable gas remains that initially present in the cavitation nuclei, and these expand and merge into predominantly vaporous, attached cavities. The void that first reaches hemispherical form determines the critical pressure connected to the globally spherical particle.

In the present experiments filtered water was seeded with almost spherical solid balls, Dynospheres, made from polymers. Three of the batches were characterized by the manufacturer (Dyno Particles AS, Norway) as hydrophobic, and two batches as hydrophilic. Hydrophilicity was obtained from functionalized monomers participating during or at the end of the polymerization, and resulting in either hydroxyl or
TABLE I. Dynospheres used for seeding in the present investigation of the tensile strength of water.

\begin{tabular}{|c|c|c|c|}
\hline $\begin{array}{l}\text { Factory } \\
\text { code }\end{array}$ & $\begin{array}{l}\text { Surface } \\
\text { character }\end{array}$ & $\begin{array}{l}\text { Base } \\
\text { material }\end{array}$ & $\begin{array}{l}\text { Diameter } \\
(\mu \mathrm{m})\end{array}$ \\
\hline EXP-SS-3.0-RXG & Hydrophobic & Polystyrene & 3.0 \\
\hline EXP-SA-3.2-RNI & Hydrophilic & $\begin{array}{l}\text { Acrylic polymer } \\
\text { with nitrate } \\
\text { groups on surface }\end{array}$ & $\sim 3.0$ \\
\hline EXP-SS-20.3-RXG & Hydrophobic & Polystyrene & 20 \\
\hline EXP-SS-42.3-RSH & Hydrophilic & $\begin{array}{l}\text { Polystyrene with hydroxyl } \\
\text { groups on surface }\end{array}$ & 30 \\
\hline EXP-SS-78.1-RXG/71 & Hydrophobic & Polystyrene & 76 \\
\hline
\end{tabular}

nitrate groups on the surface of the balls. The hydrophilic balls were delivered in water by the manufacturer, while the hydrophobic ones were delivered in a $10 \%$ sodium dodecyl sulphate-water solution to avoid clustering. The density of the balls was about $1.05 \times 10^{3} \mathrm{~kg} / \mathrm{m}^{3}$. Table I presents the manufacturer's particle characterization as well as our size measurements. Figures 1-5 show scanning electron microscopic pictures of the balls used for seeding. It should be noted that within each batch the balls are remarkably uniform, and that they are very close to being spherical, except the $3 \mu \mathrm{m}$ balls in Fig. 2, which have a major deformation from sphericity and exhibit "mountains" of dimensions up to $300 \mathrm{~nm}$. However, all the balls have planetary surface structures, most of them rather weak, of about $100 \mathrm{~nm}$ lateral extension. The balls in Figs. 4 and 5 apparently have nanoparticles of up to about 100-200 nm attached to their surface. It is characteristic that all the global radii of curvature are positive, while locally the fine scale structures also seem to exhibit negative radii of curvature (concave shape).

\section{EXPERIMENTAL SETUP}

The experimental work was carried out at the Versuchsanstalt für Wasserbau Obernach, Germany ${ }^{6}$ and tensile strength was measured with a Keller vortex-flow nozzle. ${ }^{7}$ In this apparatus the liquid rotates when it enters the nozzle so that the minimum pressure is obtained in the throat at the flow axis. This guarantees that the nozzle wall does not influence the tensile strength measured. The test rig used is

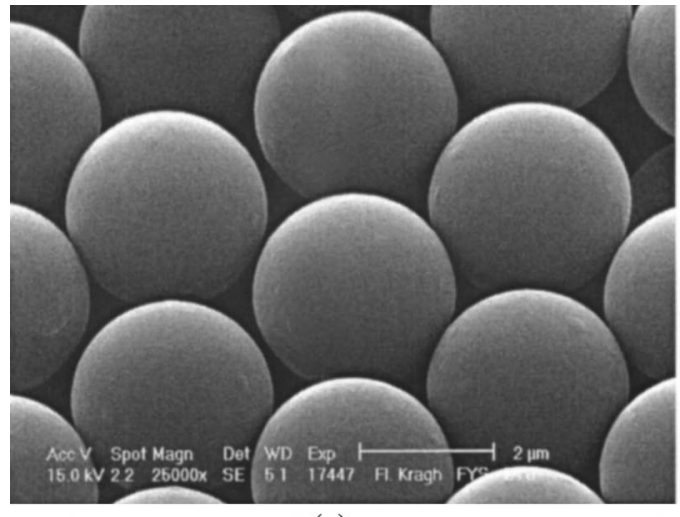

(a)

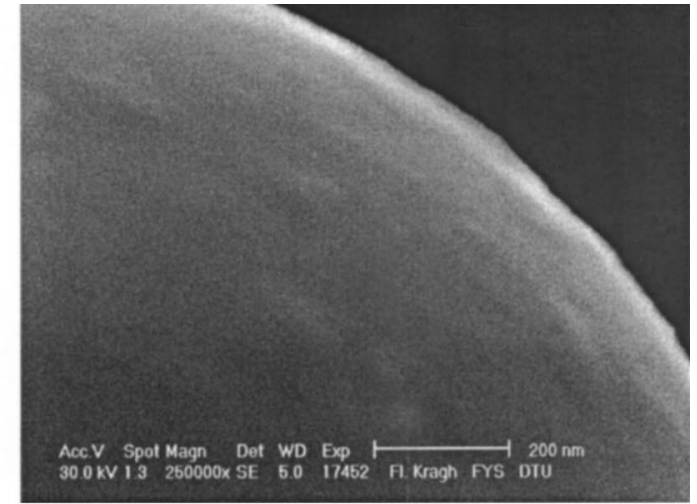

(b)

FIG. 1. The EXP-SS-3.0-RXG Dynospheres. Scale bar: (a) $2 \mu \mathrm{m}$; (b) $200 \mathrm{~nm}$. 


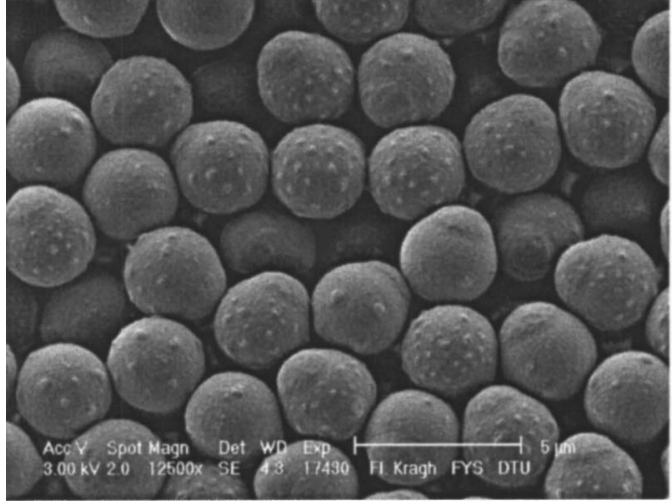

(a)

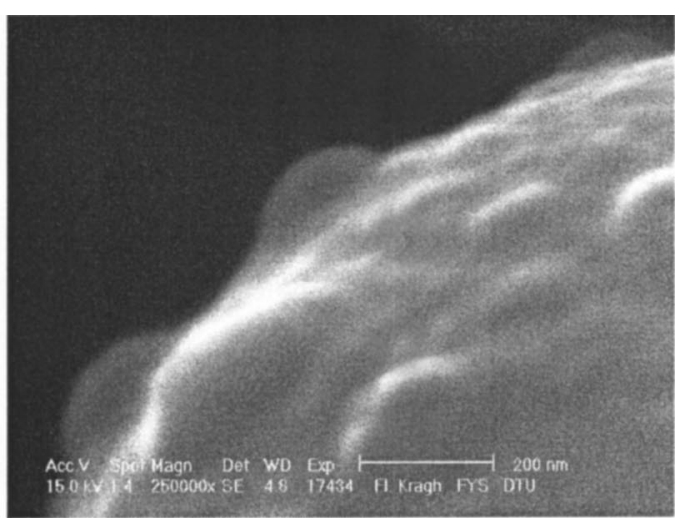

(b)

FIG. 2. The EXP-SA-3.2-RNI Dynospheres. Scale bar: (a) $5 \mu \mathrm{m}$; (b) $200 \mathrm{~nm}$.

shown schematically in Fig. 6. Tap water was supplied to the system at [1] and natural particles were filtered out in a Millipore filter [2] which was built into a 201 tank. The filter removed particles larger than about $1 \mu \mathrm{m}$. The filtered water was stored in a 301 main storage tank [3] in which the pressure was controlled by a pressurization and vacuum control unit [13]. (Gas was used to establish the pressures required, but due to the short duration of a tensile strength test, the gas content of the water was not influenced except at the very end of a test sequence.) The water temperature was kept constant with a cooling/heating system built into [3]. During experiments the valve [21] was closed and the water was led from the storage tank, where the pressure was kept at 4 bar, to the vortex-flow nozzle [5] through a mixing unit [4]. Here particles could be supplied from the $50 \mathrm{ml}$ particle storage tank [16], which was at the same pressure as [3]. The flow through [4] reduced the pressure below that in [3], so that liquid from [16] containing the particles could be mixed into the main flow. The particle flow could be controlled by the needle valve [14]. Attempts were made to measure this flow, but had to be given up due to its fast rate of change during the experiments. Therefore, no information of the rate of supply of the Dynospheres was obtained. From [5] the water passed through a flowmeter [6] and a ball valve [9], which was operated by a computer-controlled stepper motor. Dur- ing measurements of the tensile strength this valve was opened in steps to increase the flow velocity and hereby reduce the pressure at the center of the nozzle throat where the first cavitation events were to take place. If no characteristic cavitation event was recorded at a prevailing flow within a preset interval of time, the valve was opened one step further by the stepper motor, and a short interval of time was allowed, typically $0.6 \mathrm{~s}$, to achieve equilibrium in the test rig at the increased flow. Then it was again ready for detection of cavitation events.

Cavitation events were detected by two techniques: (1) by the signal from an acoustic sensor that recorded the sound pulse emitted by the implosion of each cavity when it passed into the diffusor of the vortex-flow nozzle, and (2) by the scattering of a 1-mm-diam laser beam, which was emitted from a laser diode in the upstream chamber of the nozzle and was directed along the flow axis of the vortex-flow nozzle. When the flow was noncavitating the beam was recorded by a photodetector at the nozzle axis downstream of the diffuser. The occurrence of a cavitation event at the throat axis interrupted the beam and the event was recorded. The eventsignals from each of the two transducers were sent to the computer [17], which was programmed so that a series of successive signals, typically three, from at least one of the transducers within a suitable time, typically $3 \mathrm{~s}$, was required

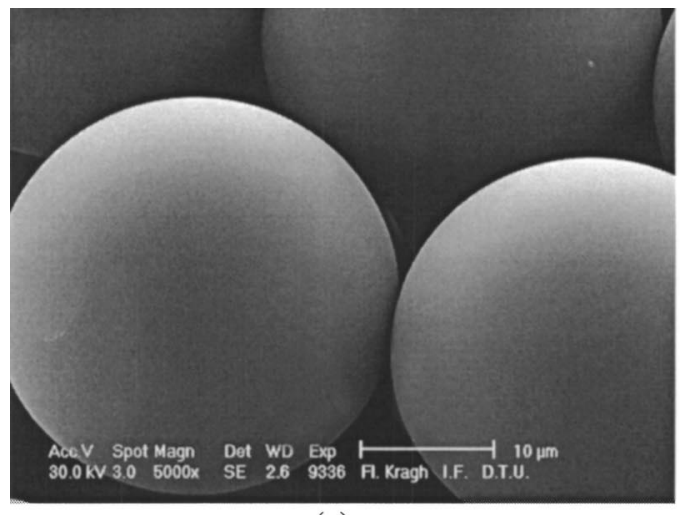

(a)

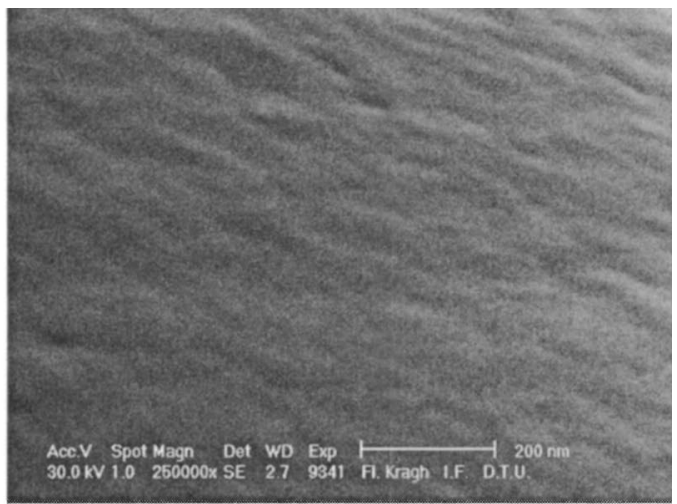

(b)

FIG. 3. The EXP-SS-42.3-RSH Dynospheres. Scale bar: (a) $10 \mu \mathrm{m}$; (b) $200 \mathrm{~nm}$. 


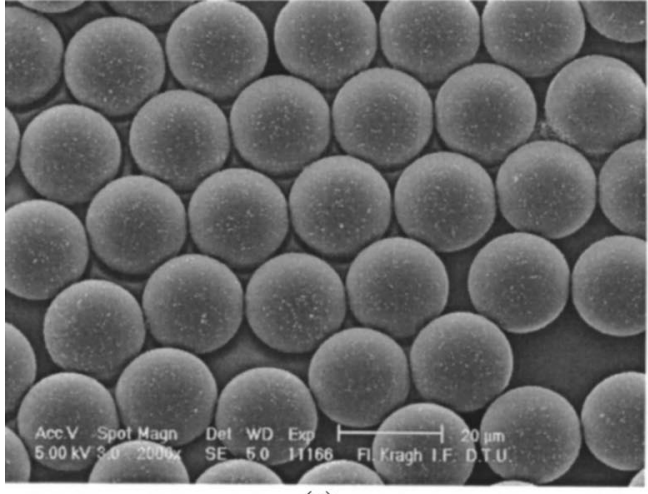

(a)

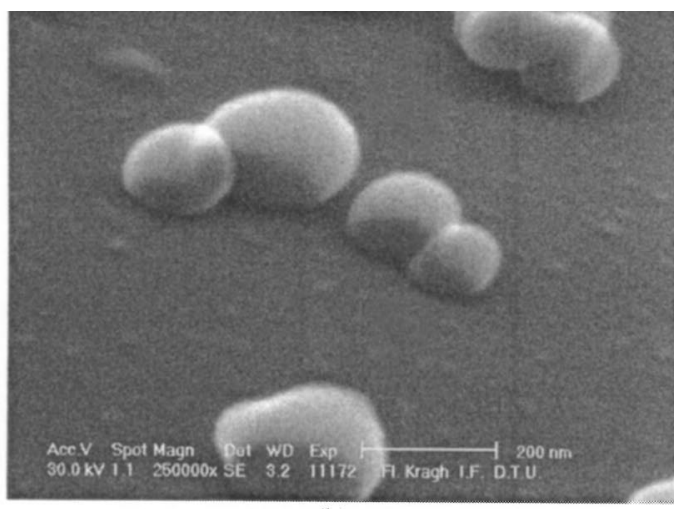

(b)

FIG. 4. The EXP-SA-20.3-RXG Dynospheres. Scale bar: (a) $20 \mu \mathrm{m}$; (b) $200 \mathrm{~nm}$.

for recognizing that cavitation events characteristic for the tensile strength of the water had occurred. In that case, the pressure, flow velocity, gas content, and temperature recorded at the previous flow step were used to calculate the tensile strength. Isolated cavitation events occurred, and may have been caused by large random nuclei. Such events were usually recorded at a tensile stress lower than the characteristic tensile strength of the liquid. However, the occurrence of a suitable number of signals within a short time, and in particular if recorded by both transducers, indicated that the tensile strength had been reached. Such groups of signals led to relatively reproducible measurements of the tensile strength. When the tensile strength was exceeded, the rate of cavitation events was in some cases so large that blocking of the nozzle flow occurred, and it strongly influenced the measurements of pressure and flow. Therefore, pressure and flow data acquired after the tensile strength had been reached led to a large dispersion of the results, and data from the preceding step were used instead. When a measurement of tensile strength had been recognized, the ball valve [9] was closed automatically, and a new experiment was performed with stepwise opening of [9] until the tensile strength was again exceeded.

Parallel to the components [5], [6], and [9] an oxygen meter [7] and a flowmeter [8] were mounted for continuous measurement of the gas content in the nozzle flow. The water that had passed through the nozzle and the oxygen meter was collected in two tanks [10] each of 131 in which the pressure was 0.2 bar, controlled by [13]. From here the water was either returned continuously to the tank [3] through the main Millipore filter [11] by use of the pump [12], or it was returned without filtering when [3] had emptied. In the latter case the valves [18]-[20] were closed, the by-pass valve [21] was opened, and the pressure control unit [13] was used to establish the reloading of [3] by maintaining 0.2 bar in [3] and applying about 0.5 bar in [10] during the transfer of water.

The vortex-flow nozzle [5] was made from glass, and thus the occurrence of cavitation could be observed visually or photographed.

\section{EXPERIMENTS}

Measurements have shown that the tensile strength of unfiltered, unpressurized tap water is very low due to the presence of random natural particles in the water. Therefore, before experiments were started the water was filtered by circulation for $2-4 \mathrm{~h}$ in the whole of the flow system at a pressure of 0.2 bar absolute. Hereby water that was cleared of particles $>1 \mu \mathrm{m}$ and of a low gas content was obtained. It was then prepressurized for $15 \mathrm{~min}$ at 6 bar. Subsequently tensile strength measurements were started, first with the fil-

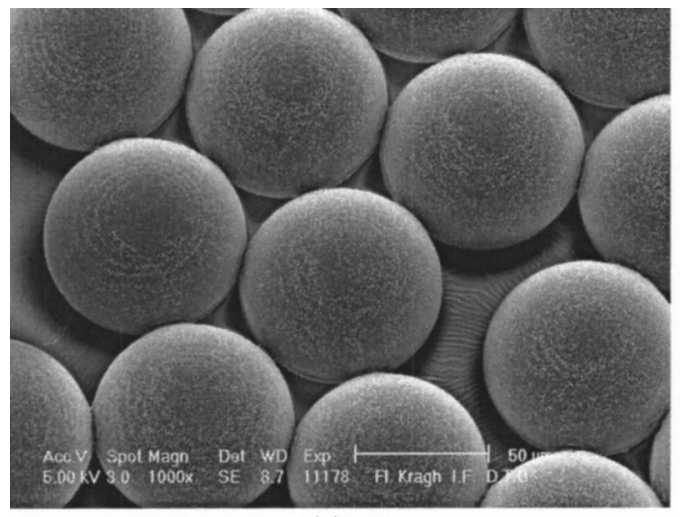

(a)

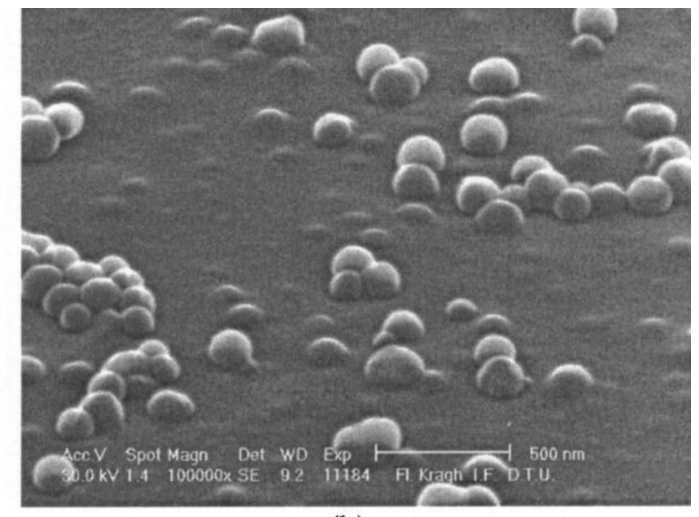

(b)

FIG. 5. The EXP-SA-78.1-RXG/71 Dynospheres. Scale bar: (a) $50 \mu \mathrm{m}$; (b) $500 \mathrm{~nm}$. 


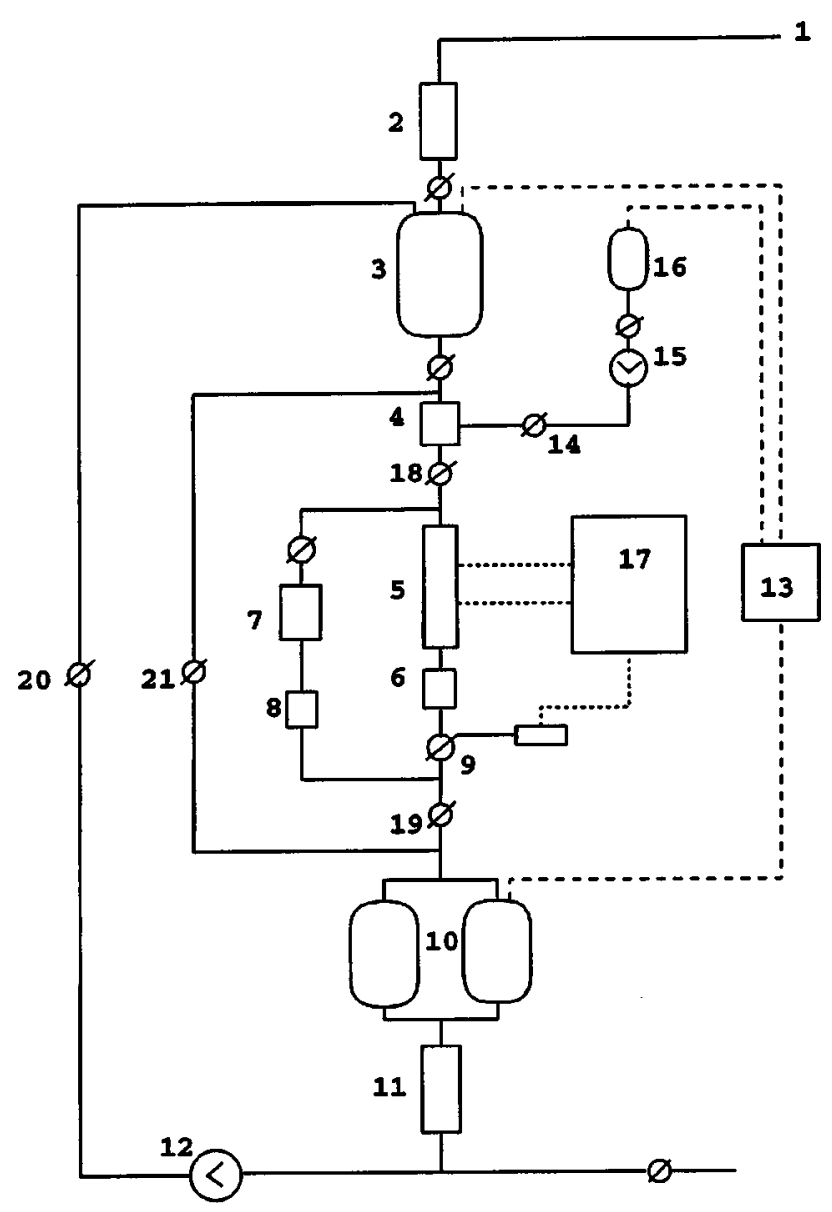

FIG. 6. Experimental setup for measurement of tensile strength. [1] Tap water supply, [2] Millipore filter, [3] mail storage tank, [4] mixing unit, [5] vortex-flow nozzle, [6] flowmeter, [7] oxygen meter, [8] flowmeter, [9] ball valve, [10] reservoir tanks, [11] main Millipore filter, [12] pump, [13] pressurization and vacuum control unit, [14] needle valve, [15] flowmeter, [16] particle storage tank, [17] computer, [18]-[21] valves.

tered water itself, the water being continuously circulated and filtered, the pressure being kept at 4 bar in the storage tank [3] and at 0.2 bar in the receiver tanks [10]. After a number of such measurements, prepressurization at 6 bar for 5 min was applied. New tensile strength measurements now generally showed increased strength. When a tensile strength of preferably not less than 1.5 bar was achieved, seeding with Dynospheres was started. A maximum tensile strength of $P_{\mathrm{ts}}=1.9$ bar was obtained for filtered water, degassed to about 0.2 bar. The water temperature was $15-18^{\circ} \mathrm{C}$ and was kept within $\pm 1{ }^{\circ} \mathrm{C}$ in each of the series of experiments.

In some cases a tensile strength was recognized from events recorded by only one of the transducers during the preset time interval. Occasionally the acoustic signals were weak, and only optical signals were recorded. These may have been caused by the passage of sufficiently large noncavitating objects, e.g., clusters or clouds of particles that triggered the optical transducer, but did not cavitate, thus leading to recognition by only this transducer of a tensile stress level lower than $P_{\text {ts }}$. In cases where only acoustic signals led to recogniton, the optical sensor may have suffered permanent blocking, or the cavitation events may have been due to particles passing the nozzle off the flow axis,

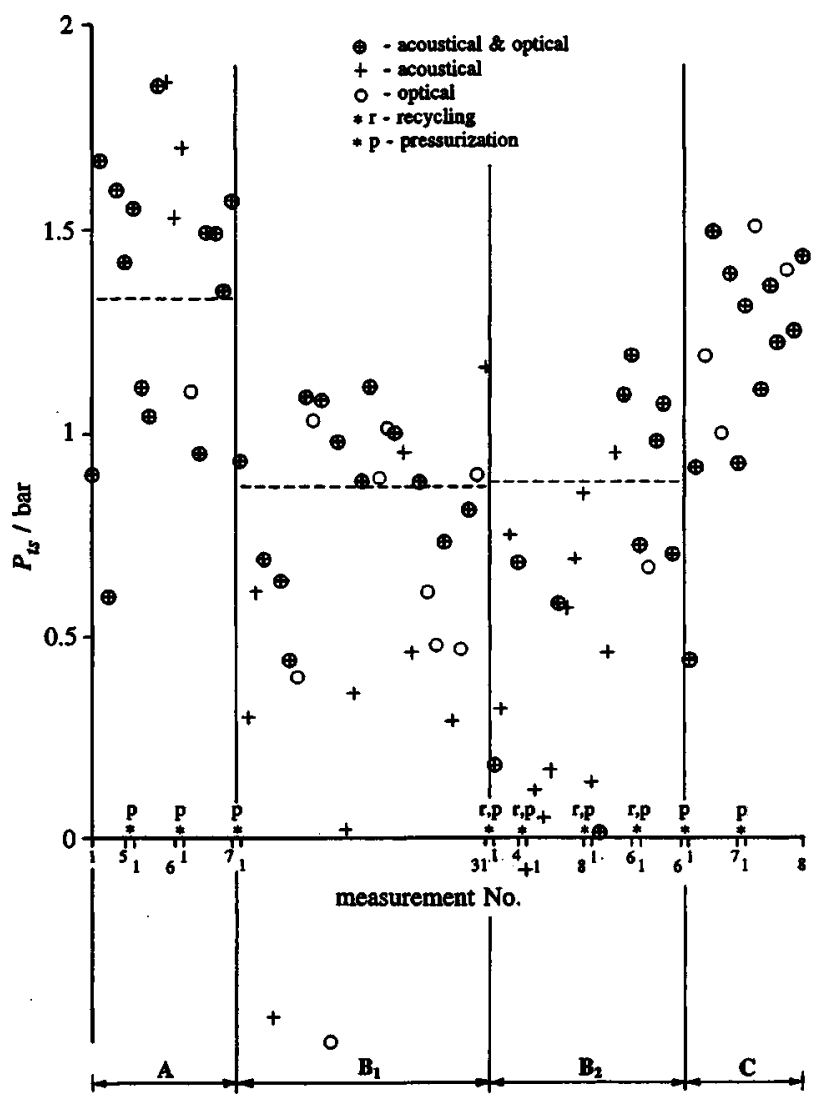

FIG. 7. Measurements of tensile strength $P_{\mathrm{ts}}$ made with the $30-\mu \mathrm{m}$-diam EXP-SS-42.3-RSH Dynospheres. (A) Filtration to clear the water of particles. $\left(\mathrm{B}_{1}\right)$ Continuous seeding with Dynospheres. $\left(\mathrm{B}_{2}\right)$ Recycling of water containing Dynospheres. (C) Filtration to clear the water of particles.

e.g., natural particles of a density notably larger than that of water.

The flow circuit with the filter [11] was used for clearing the water of particles, natural as well as seeded ones, and during measurements of its tensile strength. Due to limitations in the amount of Dynospheres available and a too fast consumption of them, it became necessary already during the first seeding experiment (with EXP-SS-42.3-RSH) to recycle the water containing the balls from [10] to [3] via the by-pass by use of the pressure control unit.

With the EXP-SS-3.0-RXG and the EXP-SA-3.2-RNI Dynospheres the large number of small particles in the water resulted in a scattering of the laser beam, irrespective of the flow, and this caused a permanent triggering of the optical cavitation event sensor. Therefore, this sensor was turned off while the $3 \mu \mathrm{m}$ balls were investigated and only the acoustic sensor was used. The tensile strength measurements recorded by that sensor were not distinguishable from those measured for the filtered water itself. It is therefore concluded that the $3 \mu \mathrm{m}$ Dynospheres, though three times the size of the largest natural particles in the water, were too small to reduce the tensile strength below the value determined by the natural particles remaining after filtration.

The results of the tensile strength measurements with the balls of diameters 30, 76, and $20 \mu \mathrm{m}$ are shown in Figs. 7, 8, and 9, where the measured tensile strength is shown versus the experiment number in each of the successive series, sepa- 


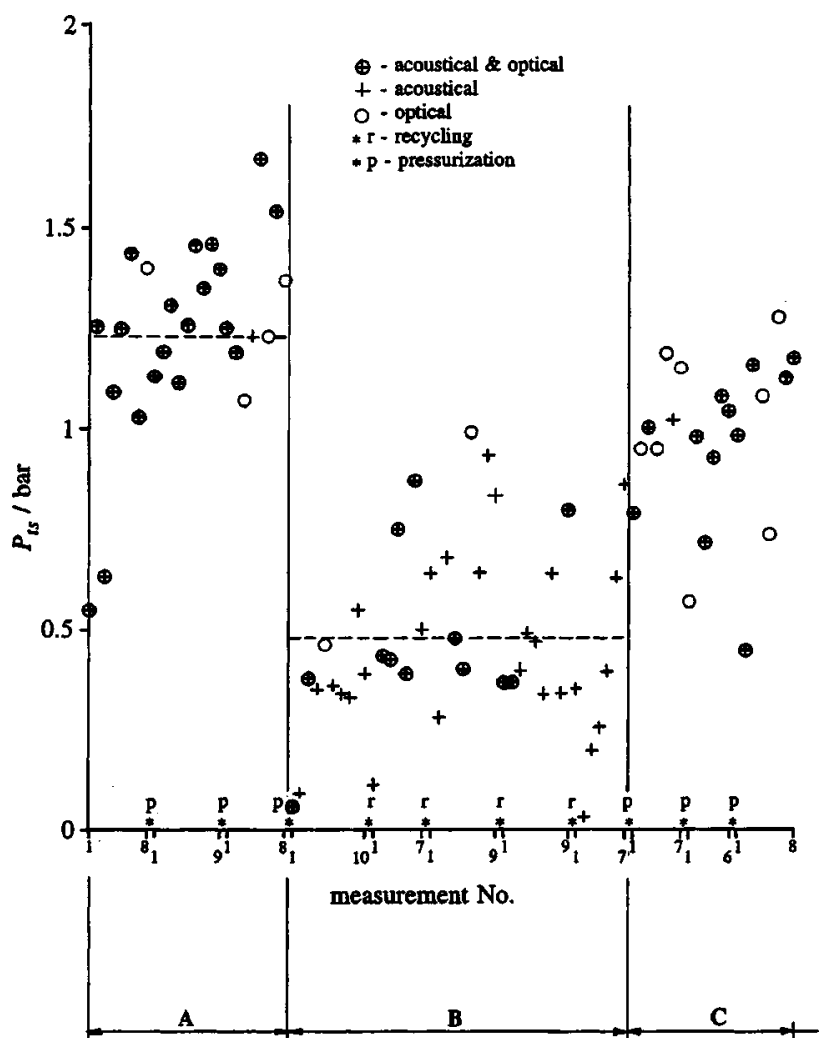

FIG. 8. Measurements of tensile strength $P_{\text {ts }}$ made with the 76- $\mu$ m-diam EXP-SS-78.1-RXG/71 Dynospheres. (A) Filtration to clear the water of particles. (B) Recycling of water containing Dynospheres. (C) Filtration to clear the water of particles.

rated by prepressurization (indicated by $p$ ) and recycling processes (indicated by $r$ ) and/or by changes in the state of seeding. The states of seeding divide the graphs into three (Figs. 8 and 9) or four (Fig. 7) major sections. Note that the first axis indicates the sequential order of the inception measurements and does not contain any time information. The time between each measurement and the processing times were very different. A whole sequence of experiments took about one day.

The first section of Figs. 7-9, (A), shows successive measurements of the tensile strength obtained during circulation and filtering of the water before addition of Dynospheres. In these measurements each of the cavitation events that was recognized as representing the tensile strength was clearly discernible (time intervals of the order of 1-2 s), i.e., there were few natural nuclei large enough to cause cavitation.

The second section of Fig. 7, $\left(B_{1}\right)$, shows the tensile strength obtained during continuous seeding with the $30 \mu \mathrm{m}$ hydrophilic Dynospheres, where a cascade of cavitation events occurred when the tensile strength was reached. The numerous cavitation events were directly visible at the nozzle throat. During the first 19 measurements the water was continuously returned to the storage tank [3] via the filter [11] and the pump [12] so that the Dynospheres were filtered out. Due to the rapid consumption of the balls, filtering had to be stopped, but seeding was continued until the tank was empty of filtered water. During this time the last 12

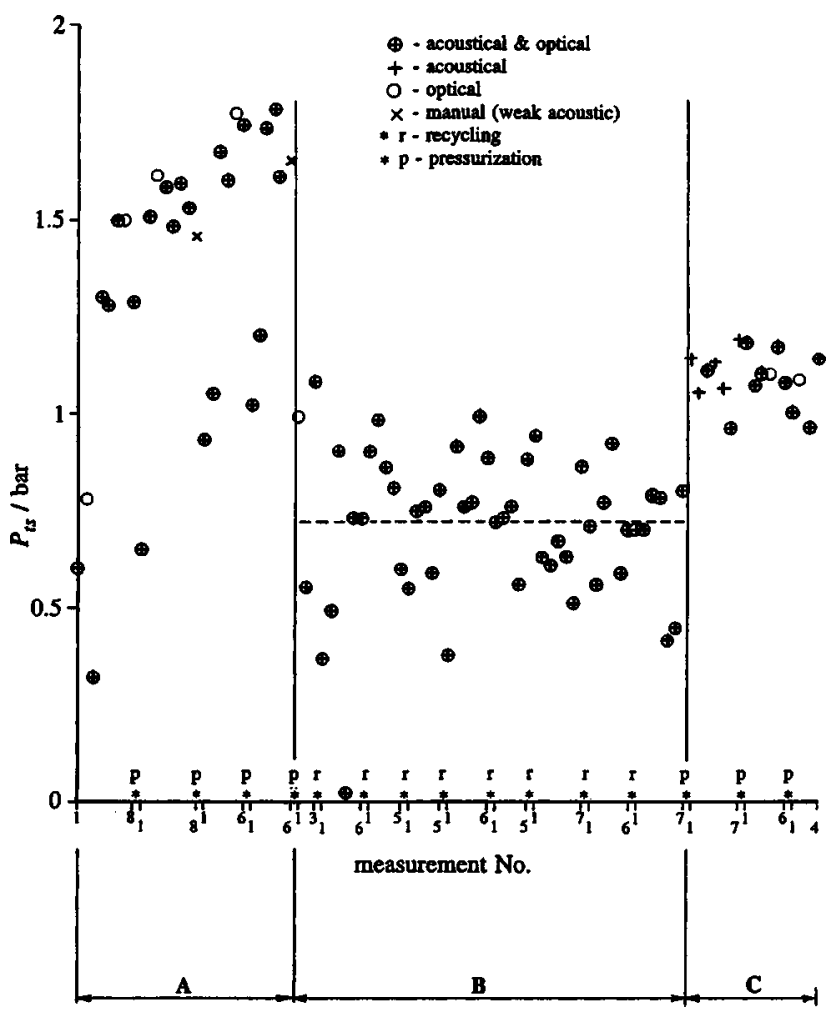

FIG. 9. Measurements of tensile strength $P_{\text {ts }}$ made with the $20-\mu \mathrm{m}$-diam EXP-SS-20.3-RXG Dynospheres. (A) Filtration to clear the water of particles. (B) Recycling of water containing Dynospheres. (C) Filtration to clear the water of particles.

measurements in Sec. $\left(B_{1}\right)$ were made. The third section of Fig. 7, $\left(\mathrm{B}_{2}\right)$, presents the measurements of tensile strength obtained after recycling and prepressurization of the water containing the Dynospheres.

The second section of Figs. 8 and 9, (B), shows the tensile strength obtained with the 76 and the $20 \mu \mathrm{m}$ hydrophobic Dynospheres, respectively. These balls were mixed directly into the water of the storage tank [3], and the experimental procedure for this section therefore corresponds to that in Sec. $\left(\mathrm{B}_{2}\right)$ of Fig. 7 , but without prepressurization in order to minimize sedimentation and clustering of the particles.

The last section in Figs. 7-9, (C), shows the tensile strength obtained when filtering was again started to remove particles from the water. It increased, but not to the level obtained in Sec. A.

For the evaluation of mean tensile strength it has been decided to include only the measurements recorded by both transducers. A few of these with very low values have been considered to be outliers caused by alien particles that have become mixed with the water in the particle storage tank or during recycling. In Table II the mean values of the tensile strength, $\left\langle P_{\mathrm{ts}}\right\rangle$, obtained with the 20,30, and $76 \mu \mathrm{m}$ Dynospheres are listed together with their uncertainty $u\left(\left\langle P_{\mathrm{ts}}\right\rangle\right)$ as well as the uncertainty of the individual measurements $u\left(P_{\mathrm{ts}}\right)$. These data are presented together with those obtained for filtered water prior to the respective seedings with Dynospheres. 
TABLE II. Mean tensile strength $\left\langle P_{\mathrm{ts}}\right\rangle$ of tap water measured first during filtering to obtain water with only natural particles $\leqslant 1 \mu \mathrm{m}$, and subsequently after seeding of the filtered water with different sizes of Dynospheres. The uncertainty $u\left(\left\langle P_{\mathrm{ts}}\right\rangle\right)$ and that of individual measurements $u\left(P_{\mathrm{ts}}\right)$ are given.

\begin{tabular}{lccc}
\hline \hline \multicolumn{1}{c}{ Diameter } & $\begin{array}{c}\text { Mean tensile } \\
\text { strength }\end{array}$ & $\begin{array}{c}\text { Uncertainty, } \\
\text { mean }\end{array}$ & $\begin{array}{c}\text { Uncertainty, } \\
\text { indiv. meas. }\end{array}$ \\
\hline \multicolumn{1}{c}{$2 R(\mu \mathrm{m})$} & $\left\langle P_{\mathrm{ts}}\right\rangle(\mathrm{bar})$ & $u\left(\left\langle P_{\mathrm{ts}}\right\rangle\right)(\mathrm{bar})$ & $u\left(P_{\mathrm{ts}}\right)(\mathrm{bar})$ \\
3.0 & $>\sim 1.5$ & & \\
$\sim 3.0$ & $>\sim 1.5$ & & \\
Nat. part. $<1$ & 1.36 & 0.08 & 0.35 \\
20 & 0.72 & 0.03 & 0.17 \\
Nat. part. $<1$ & 1.33 & 0.10 & 0.37 \\
30 Sec. $\mathrm{B}_{1}$ & 0.87 & 0.06 & 0.21 \\
30 Sec. $\mathrm{B}_{2}$ & 0.88 & 0.09 & 0.25 \\
Nat. part. $<1$ & 1.23 & 0.07 & 0.29 \\
76 & 0.48 & 0.07 & 0.24 \\
\hline \hline
\end{tabular}

\section{DISCUSSION}

\section{A. Experimental results}

In Fig. 10 the measured values of the tensile strength of water given in Table II are presented together with the curve of tensile strength $\left(P_{\mathrm{ts}}\right)_{R \text {,th }}$ corresponding to perfectly hydrophobic spheres, or vapor cavities, Eq. (1). The experimental values obtained with filtered water before seeding with Dynospheres, and thus with only natural nuclei $\leqslant 1 \mu \mathrm{m}$ are located below this curve, while those with water seeded with the almost spherical balls are located significantly above this curve. The tensile strength obtained with Dynospheres of diameters $2 R=20,30$, and $76 \mu \mathrm{m}$ can be ascribed to hemispherical vapor cavities (i.e., critical cavities) of radii $\left\langle S_{\min }\right\rangle=2.0,1.7$, and $3.0 \mu \mathrm{m}$, respectively, attached to the surface of the balls, these radii being calculated from

$$
P_{\mathrm{ts}}=2 \gamma / S_{\min }=-\left(p_{\infty}-p_{v}\right)_{\text {crit }} \text {. }
$$

They are notably smaller than the radii $R$ of the balls themselves, but on the other hand no surface structures as large as these are found on the balls. Only structures in the $100 \mathrm{~nm}$ range are characteristic, i.e., structures at least an order of size smaller than the critical radii. It is assumed that in the pressurized tanks [3] and [16], Fig. 6, gaseous interfacial nanoscale voids develop and reach dynamic equilibrium at these surface structures. Thus, on each ball numerous adjacent, nanoscale voids are supposed to exist. When a ball moves to the nozzle throat the pressure drops dramatically within a short time, and the volume of each void expands by increasing the curvature of its gas-water interface and by expanding its locus of attachment. As diffusion of gas in solution is a slow process, the content of noncondensable gas in the voids is almost constant during their expansion, and they become essentially vaporous. During growth they may merge into notably larger vaporous voids, the largest one eventually determining the tensile strength set up by the ball. The number of merging nanovoids apparently decreases with an increase of the global, positive curvature of the ball surface. As seeding the filtered water with $3 \mu \mathrm{m}$ balls caused no change to its tensile strength, it is concluded that these balls imposed a tensile strength higher than that already set by
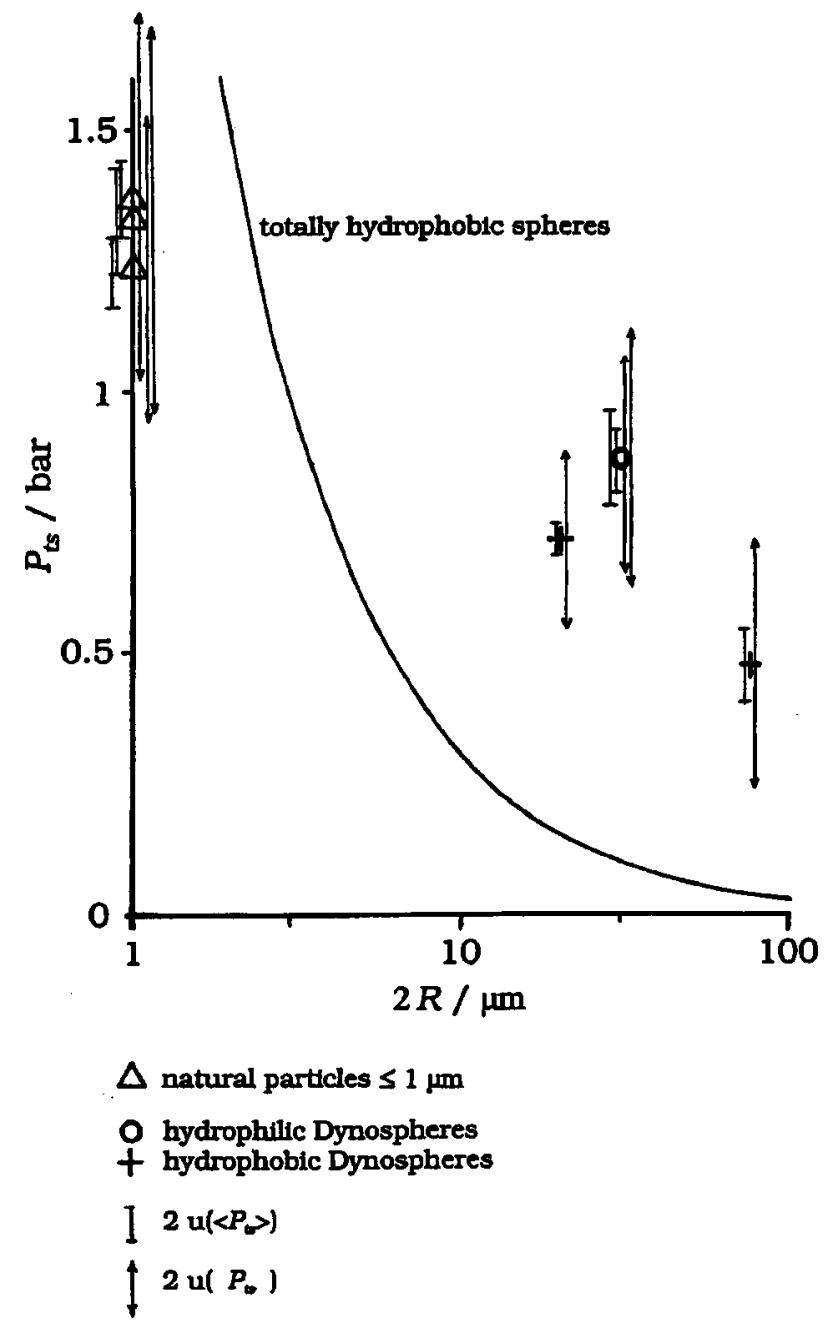

FIG. 10. The experimental mean tensile strength $\left\langle P_{\mathrm{ts}}\right\rangle$ of water filtered to remove particles $>1 \mu \mathrm{m}$, and of the water when it was subsequently seeded with Dynospheres, presented vs their diameter $2 R$. The uncertainties of the individual measurements, $u\left(P_{\mathrm{ts}}\right)$, and those of the mean values, $u\left(\left\langle P_{\mathrm{ts}}\right\rangle\right)$, are shown as error bars. Further, the curve corresponding to the theoretical tensile strength $\left(P_{\mathrm{ts}}\right)_{R \text {,th }}$ of perfectly hydrophobic spheres (or vapor cavities), Eq. (1), is shown. In each batch of Dynospheres the global radii $R$ are the same within $1 \%-2 \%$.

remaining natural particles of sizes up to $1 \mu \mathrm{m}$. The hydrophobic $20 \mu \mathrm{m}$ balls resulted in a tensile strength about 5 times that of vapor bubbles and as much as half that of the 1 $\mu \mathrm{m}$ natural particles. The hydrophobic $76 \mu \mathrm{m}$ balls produced a tensile strength at least 10 times that of vapor bubbles and $1 / 3$ that of $1 \mu \mathrm{m}$ natural particles. The tensile strength was also dependent on the hydrophilic/hydrophobic character of the ball surface: the $20 \mu \mathrm{m}$ hydrophobic balls gave a tensile strength lower than that of $30 \mu \mathrm{m}$ hydrophilic balls, which indicates that the merging of nanovoids is reduced by hydrophilicity. Though the balls were globally almost identical in each batch, the measured tensile strength of the seeded water was found to be a statistic quantity. Actually, this is to be expected because the small-scale surface structures are stochastic, and accordingly the number of nanovoids that merge will differ from one ball to the other.

In the present experiments with filtered water where natural particles of sizes above $1 \mu \mathrm{m}$ were removed, the 
upper limit of the tensile strength was less than $2.0 \mathrm{bar}-\mathrm{a}$ value which is below that corresponding to perfectly hydrophobic spheres (or vapor bubbles) of diameter $1 \mu \mathrm{m}$. This indicates that gaseous voids of notable size were attached to the remaining natural particles, allowing them to expand to a size larger than that of the particles themselves before reaching critical size.

Greenspan and Tschiegg's ${ }^{3}$ experiments with acoustic cavitation showed that the tensile strength of water increased when the size of the remaining natural particles was reduced, and it reached a maximum of $\sim 200$ bar when particles larger than $0.2 \mu \mathrm{m}$ were removed by filtration. Filtration to smaller sizes had no further effect. We can assume that in their acoustic experiments the interfacial voids causing inception grew by rectified diffusion of gas before being critical, and voids of attachment radius 4-7 nm have determined the tensile strength, i.e., voids notably smaller than the particles themselves. This indicates that the natural particles of diameter $0.2 \mu \mathrm{m}$ were dominated by positive global radii of curvature, and it suggests that natural particles change in character when decreasing from $1 \mu \mathrm{m}$ (the experiments of this paper) to $0.2 \mu \mathrm{m}$ diameter (Greenspan and Tschiegg). Actually, Greenspan and Tschiegg observed that with these small particles the gas content of the water was without influence on the tensile strength, which supports the above-presented interpretation. The ratio of the number of surface atoms to that of bulk atoms in a particle increases when the particle size is reduced. Therefore, surface tension forces become important when nanoscale particles are considered, and they favor a spherical shape. When particles are small, i.e., when they approach the $100 \mathrm{~nm}$ range, they may quite well be single crystals, a fact which also suggests a shape that is globally convex.

\section{B. Model of void development}

Experimental and computational investigations of atomically flat surfaces of platinum ${ }^{8}$ and molecularly smooth surfaces of mica ${ }^{9,10}$ show that at $300 \mathrm{~K}$ the first layer of water molecules is adsorbed to the solid surface in a solid-like structure, while the first hydration layer is strongly immobilized, though liquid-like. These layers show a distinct density oscillation normal to the solid surface. The following hydration layers exhibit notably less stabilization and within about $10 \AA$ bulk water conditions are achieved.

These results comply with investigations of solid surfaces with microscale roughness by atomic force microscopy, where force spectroscopy reveals that an interfacial tension is present in the layers of water adjacent to the solid surface. ${ }^{11}$ Therefore, at concave configurations of nonplanar surfaces we can expect that interfacial tension strains the molecular liquid bonds, and that gas molecules $\left(\mathrm{O}_{2}\right.$ and $\left.\mathrm{N}_{2}\right)$ in solution in the liquid tend to enter the strained region, thus causing the formation of a free liquid surface, or at sufficient negative curvature, the liquid bonds may break unprovoked. In contrast to this the interfacial tension strengthens the molecular bonds at convex configurations, and inhibits the access of gas molecules to the interface. This means that a concave surface shape promotes a hydrophobic surface char- acter, while a convex shape promotes a hydrophilic character. The initial voids formed in this way may grow as a result of resonance oscillations of the void-liquid interfaces. ${ }^{4}$ At liquid temperatures approaching the freezing point an augmented stabilization of the interfacial liquid is expected, and consequently also an increased interfacial tension. This explains the observed reduction of the tensile strength of water at temperatures below $10{ }^{\circ} \mathrm{C} .^{7,12}$

Ordinary surfaces with densely distributed small-scale corrugations are supposed to exhibit neighboring concave structures, which have developed interfacial voids, and they are separated by zones of maximum positive surface curvature along parts of their configuration. At exposure to tensile stress these voids expand and may get into contact at the maxima. In such cases they merge and form significantly larger voids at the prevailing tensile stress, i.e., they approach their limit of stability.

If we change a planar solid surface with small corrugations into one with a global curvature (such as the almost spherical balls in Figs. 1-5) a positive global curvature augments the local curvature at convex surface corrugations, and it reduces the local curvature at concave surface corrugations. The former influence tends to impede the merging of neighboring voids, while the latter tends to oppose the initial void formation. Thus, both effects increase the tensile strength of a liquid containing particles of positive global radius of curvature-the more the smaller the radius is, just as found in the experimental investigations with almost spherical balls. On the other hand, merging is facilitated if the global radius of curvature becomes negative, and a reduced tensile strength is achieved. Large natural particles commonly exhibit extended areas of negative global radius of curvature. This explains the low tensile strength of plain water, which contains large natural particles of highly irregular shape. ${ }^{5}$ Here the voids may grow to an appreciable volume that contains gas, and the tensile strength may be reduced below that of perfectly hydrophobic spheres of size similar to that of the particles.

It is conjectured that in the present experiments the $3 \mu \mathrm{m}$ almost spherical balls were small enough to prevent merging of nanoscale voids into microscale voids. Thus, the tensile strength of the seeded water remained determined by the notably smaller, but irregular natural particles. The larger balls used in the present experiments had too large global radii of curvature to prevent the merging of considerable numbers of adjacent nanovoids connected to the surface structures of the almost spherical balls. Therefore, critical microvoids developed, notably smaller than the balls themselves, but larger than those characteristic of the remaining natural particles.

\section{ACKNOWLEDGMENTS}

The authors wish to thank the Norwegian Research Foundation for financial support under Grant No. 119167/ 431, which has made the present experimental study possible. We also wish to express our gratitude to Dyno Particles AS, Norway (now Dynal Biotech) for generously supplying the Dynospheres forming the basis of the project, and to 
Danfoss A/S, Denmark for lending us equipment for flow measurement. Finally, we are obliged to Flemming Kragh for specimen preparation and scanning electron microscopy of the Dynospheres used in the experimental work. We thank Dr. Jette Mackintosh for assistance in preparation of the manuscript and the Quantum Protein Center, DTU for secretarial help.

${ }^{1}$ P. S. Epstein and M. S. Plesset, "On the stability of gas bubbles in liquidgas solutions," J. Chem. Phys. 18, 1505 (1950).

${ }^{2}$ E. N. Harvey, D. K. Barnes, W. D. McElroy, A. H. Whiteley, D. C. Pease, and K. W. Cooper, "Bubble formation in animals. I. Physical factors," J. Cell. Comp. Physiol. 24, 1 (1944).

${ }^{3}$ M. Greenspan and C. E. Tschiegg, "Radiation-induced acoustic cavitation: Apparatus and some results," J. Res. Natl. Bur. Stand., Sect. C 71, 299 (1967).

${ }^{4}$ K. A. Mørch, "Cavitation nuclei and bubble formation-A dynamic liquid-solid interface problem," J. Fluids Eng. 122, 494 (2000).

${ }^{5}$ L. A. Crum, "Tensile strength of water," Nature (London) 278, 148 (1979).
${ }^{6} \mathrm{H}$. B. Marschall, "Hohlraumbildung in Wasser an FestkörperGrenzflächen als Kavitations-keime," Diplomarbeit, Versuchsanstalt für Wasserbau und Wassermengenwirtschaft der Technischen Universität München/Obernach, Germany.

${ }^{7}$ A. P. Keller, Schlussbericht über das Forschungsvorhaben "Beginnende Kavitation, Zugspannungen in Flüssigkeiten," 2. Teil, Versuchsanstalt für Wasserbau und Wassermengenwirtschaft der Technischen Universität Müchen/Obernach, Germany.

${ }^{8}$ X. Xia, L. Perera, U. Essmann, and M. L. Berkowitz, "The structure of water at platimum/water interfaces, molecular dynamics computer simulations," Surf. Sci. 335, 401 (1995).

${ }^{9}$ L. Cheng, L, P. Fenter, K. L. Nagy, M. L. Schlegel, and N. C. Sturgchio, "Molecular-scale density oscillations in water adjacent to a mica surface," Phys. Rev. Lett. 87, 156103 (2001).

${ }^{10}$ S.-H. Park and G. Sposito, "Structure of water adsorbed on a mica surface," Phys. Rev. Lett. 89, 085501 (2002).

${ }^{11}$ N. A. Mortensen, A. Kühle, and K. A. Mørch, "Interfacial tension in water at solid surfaces," Proceedings of the Third International Symposium on Cavitation, Grenoble, France, edited by J. M. Michel and H. Kato, Vol. 1, pp. 87-91.

${ }^{12}$ L. J. Briggs, "Limiting negative pressure of water," J. Appl. Phys. 21, 721 (1950) 